\title{
Análisis del proceso de recuperación de la batería de alta tensión del vehículo Toyota Highlander Híbrido
}

\section{Analysis of the recovery process of the high tension battery of the Toyota Highlander Hybrid vehicle}

Daniela Alexandra Jerez Mayorga

Universidad Internacional del Ecuador, Ecuador

Edison Andrés Ayala Ortiz

Universidad Internacional del Ecuador, Ecuador

Edwin Giovanny Puente Moromenacho

Universidad Internacional del Ecuador, Ecuador

Autor para correspondencia: djerez@uide.edu.ec, aayala@uide.edu.ec,epuente@uide.edu.ec Fecha de recepción: 23 de junio de 2018 - Fecha de aceptación: 27 de agosto de 2018

Resumen: El porcentaje de vehículos híbridos en el país ha crecido considerablemente desde su aparición en el año 2009, y si con los mecanismos convencionales, tener un vehículo en perfectas condiciones era la principal preocupación de muchos usuarios esta inquietud se multiplica en quienes poseen un vehículo híbrido o eléctrico, ya que se desconoce sobre su sistema de batería de alto voltaje y su tiempo de vida útil, sin embargo los fabricantes de estos vehículos hablan de garantías que se cubren al adquirir un modelo amigable al ecosistema, y tambien ofrecen el servicio de cambio de batería completa cuando esta haya terminado su tiempo de vida útil, pero puede bordear unos cuantos miles de dólares en el mercado nacional y al no contar con este capital se verán en la necesidad de encontrar una solución más económica y confiable. A continuación, se presenta el proceso de recuperación de la batería híbrida del Toyota Highlander Híbrido a partir de la extracción del paquete de baterías, el análisis del estado de cada una de sus celdas a través del uso de un banco comprobador, para así conocer cuáles son las celdas que están en mal estado causando fallos en el funcionamiento del vehículo.

Palabras Claves: híbrido; batería; recuperación; carga; ahorro

\begin{abstract}
The percentage of hybrid vehicles in the country has grown considerably since its appearance in 2009, and if with conventional mechanisms, having a vehicle in perfect condition was the main concern of many users this concern is multiplied in those who own a hybrid vehicle or electric, as it is unknown about its high-voltage battery system and its useful life, however the manufacturers of these vehicles talk about guarantees that are covered when acquiring a model friendly to the ecosystem, and also offer the service of change of full battery when it has finished its useful life, but can border a few thousand dollars in the domestic market and by not having this capital will be in the need to find a more economical and reliable solution. Next, the process of recovering the hybrid battery of the Toyota Highlander Hybrid is presented from the extraction of the battery pack, the analysis of the state of each of its cells through the use of a tester bank, in order to know which are the cells that are in poor condition causing malfunctions in the vehicle
\end{abstract}

Key words: hybrid; battery; recovery; charge; saving 


\section{Introducción}

Los índices de contaminación mundial preocupan a las grandes industrias quienes hoy en día optan por fabricar productos que al usarse emitan menos gases nocivos al ecosistema, y la industria automotriz no puede ser indiferente ante esta situación a la que también se suma la cantidad finita de recursos en pozos petrolíferos que tarde o temprano terminará por agotar la fuente principal de combustible útil en el automóvil, creándose de esta manera nuevas alternativas para la movilidad automovilística. Es así como en el mercado mundial existen algunas marcas que apuestan a la fabricación de autos con tecnología futuristas como vehículos híbridos o eléctricos, como son Toyota, Kìa, Chevrolet, entre otras.

En 1997 Toyota Motor Corporation lanza al mercado el Prius como primer modelo híbrido y se vendieron cerca de 6 millones de vehículos híbridos a nivel mundial (Toyota, 2018), esta cifra ha ido creciendo día a día hasta superar los 10 millones de unidades vendidas en todos sus modelos híbridos en enero de 2017 (Pèrez, 2017). Más que un hito numérico, esto demuestra el poder de permanencia de una tecnología que ahora está emergiendo como una solución principal para reducir las emisiones de gases de efecto invernadero y otros contaminantes.

En la figura 1 se muestra el número de ventas de vehículos híbridos en Ecuador entre los años 2010 y 2016, tiempo en el cual tenemos 11.552 unidades vendidas de las cuales 4.843 unidades corresponden a la marca Toyota, lo que demuestra que es líder en el mercado ya que abarca el $41.92 \%$ de ventas aproximadamente, durante el período mencionado.

\begin{tabular}{|c|c|c|c|c|c|c|c|c|c|c|c|c|c|c|}
\hline \multicolumn{15}{|c|}{ VENTAS DE VEHÍCULOS HÍBRIDOS POR MARCA } \\
\hline \multicolumn{15}{|c|}{ En unidades / porcentaje de participación 2010-2016 } \\
\hline MARCA & 2010 & $\%$ & 2011 & $\%$ & 2012 & $\%$ & 2013 & $\%$ & 2014 & $\%$ & 2015 & $\%$ & 2016 & $\%$ \\
\hline HYUNDAI & & $0.00 \%$ & - & $0.00 \%$ & - & $0.00 \%$ & - & $0,00 \%$ & 470 & $52,69 \%$ & 717 & $78,88 \%$ & 645 & $65.09 \%$ \\
\hline TOYOTA & 1840 & $40,81 \%$ & 557 & $23.91 \%$ & 1352 & $96,57 \%$ & 417 & $80,04 \%$ & 304 & $34,08 \%$ & 155 & $17,05 \%$ & 218 & $22,00 \%$ \\
\hline $\mathrm{KIA}$ & - & $0,00 \%$ & - & $0.00 \%$ & - & $0.00 \%$ & - & $0.00 \%$ & 74 & $8.30 \%$ & 20 & $2,20 \%$ & 123 & $12,41 \%$ \\
\hline PORSCHE & 54 & $1,20 \%$ & 111 & $4,76 \%$ & 25 & $1.79 \%$ & 1 & $0.19 \%$ & 4 & $0.45 \%$ & 7 & $0.77 \%$ & 3 & $0.30 \%$ \\
\hline HONDA & - & $0.00 \%$ & - & $0.00 \%$ & - & $0.00 \%$ & - & $0.00 \%$ & 1 & $0.11 \%$ & 2 & $0.22 \%$ & 2 & $0.20 \%$ \\
\hline BMW & 154 & $3.42 \%$ & 82 & $3.52 \%$ & 5 & $0.36 \%$ & - & $0.00 \%$ & 23 & $2,58 \%$ & 3 & $0.33 \%$ & - & $0.00 \%$ \\
\hline AUDI & - & $0.00 \%$ & - & $0.00 \%$ & - & $0.00 \%$ & - & $0.00 \%$ & 7 & $0.78 \%$ & - & $0,00 \%$ & - & $0.00 \%$ \\
\hline FORD & 1056 & $23,42 \%$ & 1034 & $44,38 \%$ & 3 & $0.21 \%$ & 33 & $6,33 \%$ & 2 & $0.22 \%$ & 3 & $0.33 \%$ & - & $0.00 \%$ \\
\hline VOLKSWAGEN & - & $0.00 \%$ & - & $0.00 \%$ & - & $0.00 \%$ & - & $0.00 \%$ & 1 & $0.11 \%$ & - & $0.00 \%$ & - & $0.00 \%$ \\
\hline NISSAN & - & $0,00 \%$ & - & $0.00 \%$ & - & $0.00 \%$ & - & $0.00 \%$ & 4 & $0.45 \%$ & - & $0.00 \%$ & - & $0.00 \%$ \\
\hline LEXUS & 500 & $11,09 \%$ & 59 & $2,53 \%$ & 2 & $0.14 \%$ & 6 & $1.15 \%$ & 2 & $0.22 \%$ & 2 & $0,22 \%$ & - & $0.00 \%$ \\
\hline CHEVROLET & 711 & $15,77 \%$ & 328 & $14,08 \%$ & 3 & $0.21 \%$ & 1 & $0.19 \%$ & - & $0.00 \%$ & - & $0.00 \%$ & - & $0.00 \%$ \\
\hline MERCEDES BENZ & 86 & $1,91 \%$ & 78 & $3.35 \%$ & - & $0.00 \%$ & - & $0.00 \%$ & - & $0.00 \%$ & - & $0.00 \%$ & - & $0.00 \%$ \\
\hline GMC & 53 & $1,18 \%$ & 13 & $0.56 \%$ & 3 & $0.21 \%$ & - & $0.00 \%$ & - & $0.00 \%$ & - & $0.00 \%$ & - & $0.00 \%$ \\
\hline OTRAS & 55 & $1,22 \%$ & 68 & $2,92 \%$ & 7 & $0,50 \%$ & 63 & $12,09 \%$ & - & $0.00 \%$ & - & $0.00 \%$ & - & $0.00 \%$ \\
\hline TOTAL & 4509 & $100,00 \%$ & 2330 & $100,00 \%$ & 1400 & $100.00 \%$ & 521 & $100,00 \%$ & 892 & $100.00 \%$ & 909 & $100,00 \%$ & 991 & $100.00 \%$ \\
\hline
\end{tabular}

Figura 1. Ventas nacionales de vehículos híbridos por marca (AEADE, 2017)

Con estos datos se demuestra que el mejor momento para la empresa japonesa fue en el año 2010 donde alcanzó la cúspide en ventas, las mismas que han ido disminuyendo por una serie de factores como el cambio y aumento de impuestos sobre estos vehículos especialmente a los de mayor cilindrada a más de eso se sumaron nuevas marcas que lanzaron sus modelos híbridos al mercado como Hyundai y Kia, cerrando el año 2016 como segundo en el mercado.

\section{Fundamento teórico}




\section{Toyota's hybrid synergy drive (HSD)}

El sistema Hybrid Synergy Drive de Toyota se compone de: un motor de gasolina, un motor eléctrico, un generador eléctrico, la unidad de control y un dispositivo dividido que utiliza un tipo especial de caja de engranajes para distribuir suavemente la potencia del motor de combustión interna y el motor eléctrico y su batería de alta tensión. (Toyota, Blog Toyota, 2015).

Como sistema completo, Hybrid Synergy Drive es una tecnología inteligente y de ahorro de combustible que puede alternar de forma automática y sin interrupciones entre la potencia eléctrica y la potencia del motor convencional. Capaz de adaptarse a diferentes condiciones de manejo, controla de forma inteligente la energía proveniente de ambas fuentes y comunica al automóvil cómo combinarlas para obtener la mayor eficiencia y rendimiento.

\section{Componentes del hybrid synergy drive de Toyota} de Toyota.

En la siguiente figura se muestra todos los componentes principales del sistema híbrido

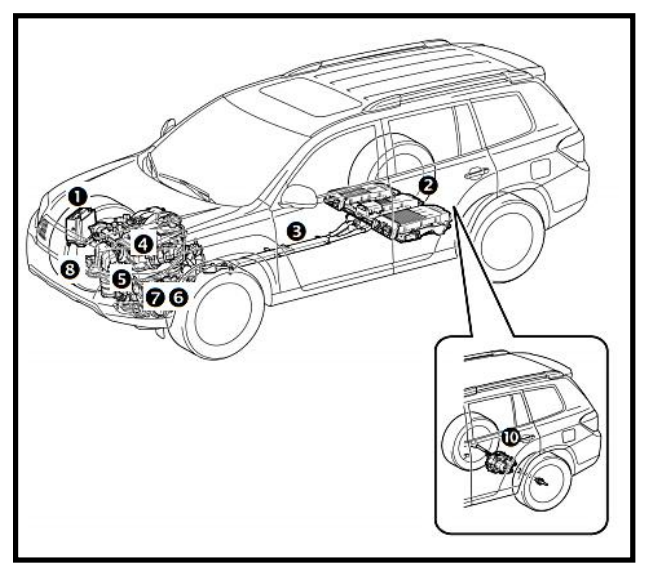

Figura 2. Ubicación de los componentes del sistema híbrido

(Toyota, 2008)

Tabla 1. Descripción de los componentes principales del sistema híbrido (Toyota, 2008)

\begin{tabular}{|c|c|c|}
\hline & Componente & Descripción \\
\hline 1 & $\begin{array}{l}\text { Batería auxiliar de } 12 \\
\text { voltios }\end{array}$ & Una batería de plomo-ácido suministra energía a los dispositivos de bajo voltaje \\
\hline 2 & Batería de alta tensión & $\begin{array}{l}\text { Un paquete de baterías de hidruro metálico de níquel de } 288 \text { voltios que consta } \\
\text { de } 30 \text { módulos de } 9,6 \text { voltios de bajo voltaje conectados en serie }\end{array}$ \\
\hline 3 & Cables de alto voltaje & $\begin{array}{l}\text { Los cables de alimentación de color naranja transportan corriente directa de alto } \\
\text { voltaje entre el paquete de baterías de alta tensión, el inversor / convertidor } \\
\text { también convierte la electricidad de C.A. del generador eléctrico y los motores } \\
\text { eléctricos (frenado regenerativo) }\end{array}$ \\
\hline 4 & Inversor/Convertidor & $\begin{array}{l}\text { Aumenta e invierte la electricidad de alto voltaje del paquete de baterías de alto } \\
\text { voltaje a la electricidad trifásica de corriente alterna que impulsa los motores } \\
\text { eléctricos. El inversor/convertidor también convierte la corriente eléctrica de } \\
\text { corriente alterna del generador eléctrico y el frenado regenerativo a corriente } \\
\text { continua que recarga el paquete de batería de alta tensión. }\end{array}$ \\
\hline
\end{tabular}




\begin{tabular}{|c|c|c|}
\hline \multirow[t]{4}{*}{5} & Motor de combustión & Proporciona dos funciones \\
\hline & interna & I. Transmite potencia a las ruedas \\
\hline & & Transmite potencia al generador \\
\hline & & $\begin{array}{l}\text { El motor se enciende y se detiene bajo el control de la computadora del } \\
\text { vehículo. }\end{array}$ \\
\hline 6 & Motor eléctrico frontal & $\begin{array}{l}\text { Motor trifásico de corriente alterna de imán permanente ubicado en el eje } \\
\text { delantero y es usado para trasmitir potencia a las ruedas delanteras. }\end{array}$ \\
\hline 7 & Generador eléctrico & $\begin{array}{l}\text { Generador trifásico de corriente alterna encargado de recargar la batería de alto } \\
\text { voltaje. }\end{array}$ \\
\hline 8 & $\begin{array}{l}\text { Compresor } \mathrm{A} / \mathrm{C} \text { con } \\
\text { inversor }\end{array}$ & A/C moto compresor manejado eléctricamente. \\
\hline \multirow[t]{2}{*}{9} & Tanque de combustible y & El tanque almacena combustible y provee gasolina al motor a través de las \\
\hline & líneas de combustible & líneas de combustible ubicadas bajo el centro del vehículo. \\
\hline 10 & Motor eléctrico posterior & $\begin{array}{l}\text { Motor trifásico de corriente alterna de imán permanente ubicado en el eje } \\
\text { posterior y es usado para trasmitir potencia a las ruedas posteriores. }\end{array}$ \\
\hline
\end{tabular}

\section{Toyota Highlander Híbrido}

\section{Materiales y métodos}

Fue uno de los primeros crossovers en ofrecer una variante híbrida, y ha llegado a su tercera generación, luego de que su primer lanzamiento fuera en el 2005 con un modelo 2006 con un motor V-6 de 3.3 litros acoplado a un motor eléctrico para una salida total del sistema de $268 \mathrm{hp}$. La producción de Highlander híbrido de la primera generación finalizó para el año modelo 2007.

El Highlander híbrido de segunda generación debutó como un modelo 2008 con el mismo tren motriz que el modelo saliente, pero se ha ajustado hasta $270 \mathrm{hp} \mathrm{combinados.} \mathrm{Toyota}$ actualizó el Highlander Híbrido para el año modelo 2011 con un nuevo motor V-6 de 3.5 litros del Lexus RX 450h, aumentando la producción total a 280 hp.

Toyota introdujo el Toyota Highlander Híbrido de tercera generación como modelo 2014 en 2013 con el mismo 3.5 litros V-6 acoplado a un motor eléctrico. Para la actualización del año modelo 2016, Toyota agregó un nuevo V-6 de 3.5 litros de inyección directa que aumenta la producción total a $306 \mathrm{hp}$. (Motor trend, 2008) 


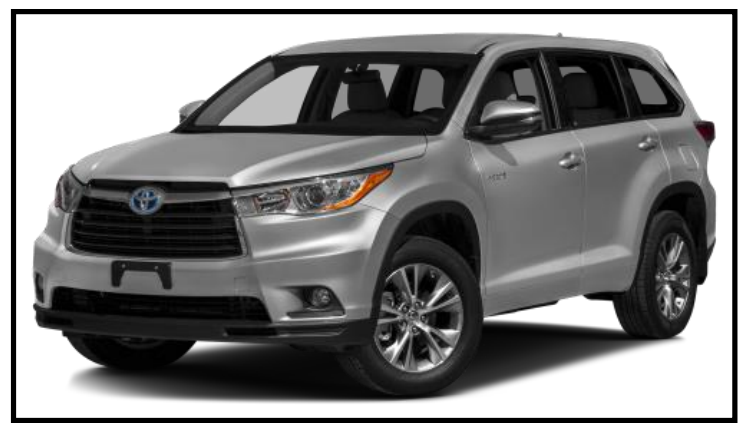

Figura 4. Toyota Highlander 2014

(Cars, 2014)

Batería de alta tension del Toyota Hignlander Híbrido

Las baterías híbridas para automóvil consisten en muchas celdas conectadas en serie y empacadas en un contenedor. Estos se llaman módulos, monitoreado diversos puntos importantes tales como la refrigeración, la calefacción, el voltaje, amperaje, la detección de fugas de alto voltaje, además de incluir los relés para garantizar una vida larga y segura.

La batería de alta tensión del Toyota Highlander Híbrido están fabricadas de:

- Ánodo (+): Oxido de Níquel

- Cátodo (-): Hidruro Metálico

- Electrolito de hidruro de potasio

Una batería de NiMH que quede al 100\% de estado de carga (SOC-State of charge) se descargará a una velocidad mayor que una a 40\% SOC. Cuanto más bajo es el SOC, más lentas son las reacciones químicas; así que cuanto más lentas sean las reacciones químicas, más durara. La menor temperatura ralentiza tanto las reacciones químicas como la tasa de autodescarga, por lo que las temperaturas más frías son mejores para el almacenamiento que las más cálidas. (Curtis Anderson, 2010)

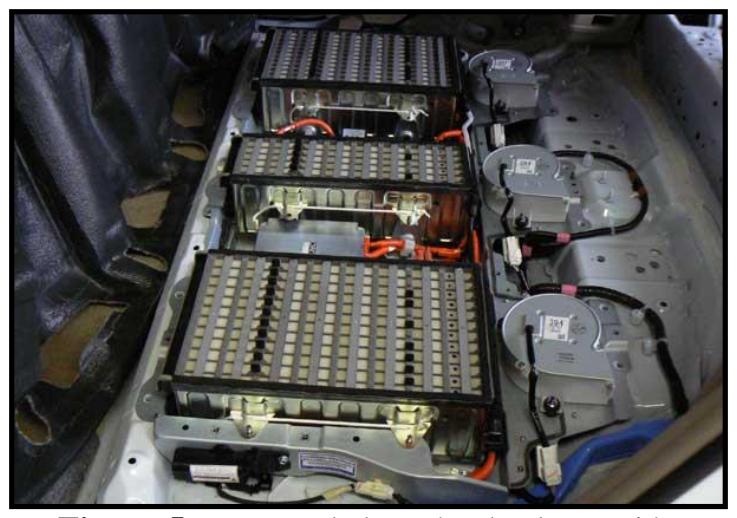

Figura 5. Paquete de baterías de alta tensión (Ecolectric Battery, 2017) 
Tabla 2. Datos generales del paquete de alta tension (Toyota, 2008)

\begin{tabular}{lc}
\hline \multicolumn{2}{c}{ Paquete de batería de alta tensión } \\
\hline Voltaje del paquete de baterías & $288 \mathrm{~V}$ \\
Cantidad de módulos en el paquete de baterías & 30 \\
Voltaje del módulo de la batería & $9.6 \mathrm{~V}$ \\
Dimensiones del módulo de la batería & $18.5 \times 382 \times 86 \mathrm{~mm}$ \\
Peso del modulo & $1.5 \mathrm{Kg}$ \\
Dimensiones del paquete de baterías & $630 \times 1080 \times 180 \mathrm{~mm}$ \\
Peso del paquete de batería & $69 \mathrm{Kg}$ \\
\hline
\end{tabular}

\section{Charger Research}

El banco de trabajo "Charger Research" es un equipo que permite evaluar el estado, funcionamiento y también permite generar cargas y descargas de las baterías que vayamos a conectar. Las baterías que puede evaluar son baterías de carros híbridos y eléctricos; la unidad está en posibilidades de reparar las celdas internas de la batería.

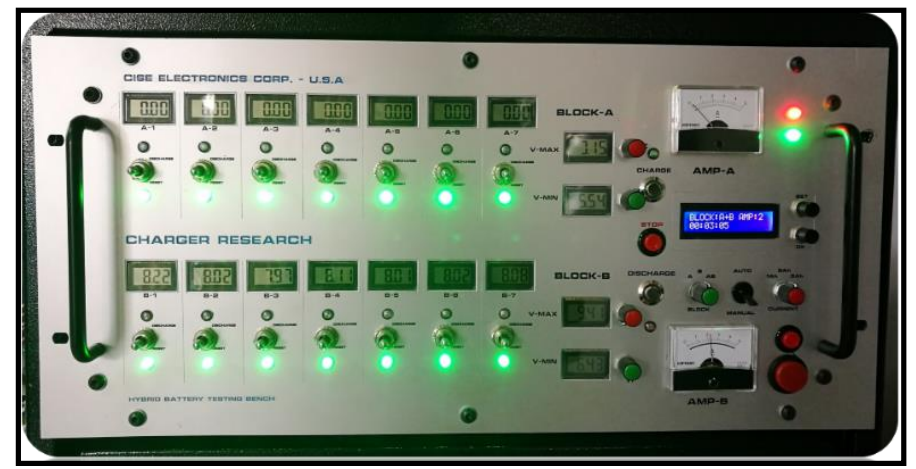

Figura 6. Panel de control del Banco de Pruebas para baterías híbridas (TAAET Electronics, 2017)

\section{Función}

El banco de pruebas Charger Research por motivos de seguridad tiene dos bloques por separados nombrados como bloque A y B. Por cada uno de los bloques se pueden conectar hasta siete celdas de baterías con una tensión nominal de $7.2 \mathrm{~V}$, el voltaje total no supera el valor de sesenta voltios para que no implique ningún daño para el usuario por exceso de voltaje.

De esta forma con los dos bloques se puede evaluar hasta 14 celdas al mismo momento. En cada celda que sea analizar, cargar o descargar posee un medidor individual de voltaje. Cada recuperación conlleva a una carga y descarga para cada celda con esto se analiza la corriente y tensión por medio de dos medidores para verificar los voltajes mínimos como el máximo de cada celda dependiendo a los voltajes que estén configurados en el equipo para descarga y carga de cada celda; cada bloque se configura con dos celdas por bloque. Cada bloque opera por separado el A o el B trabajan por separados.

\section{Partes}




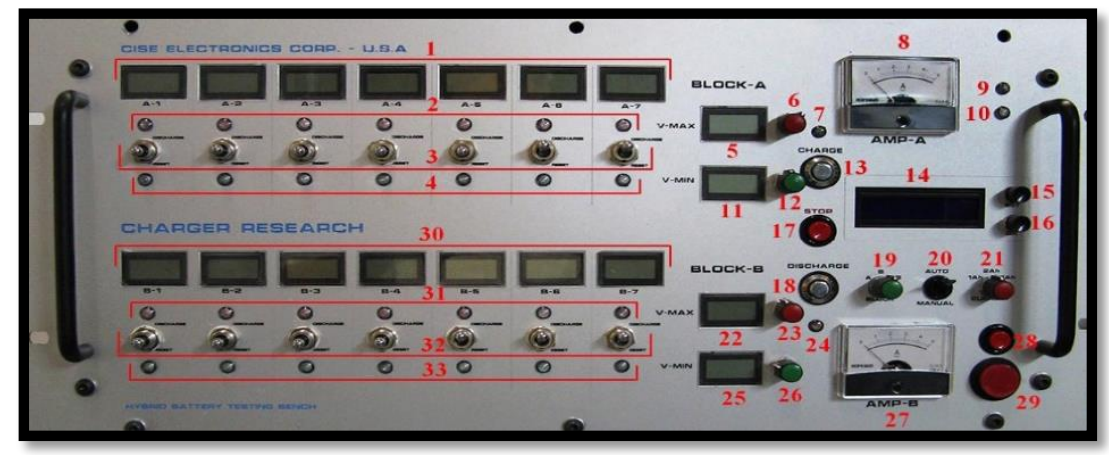

Figura 7. Partes del banco comprobador de baterías hibridas

(TAAET Electronics , 2017)

El banco de pruebas para baterías híbridas consta de 33 partes que se describen a continuation:

Tabla 3. Partes del Charger Research (TAAET Electronics , 2017)

Partes del Charger Research

1 Conjunto voltímetros bloque A

2 Conjunto leds indicación voltaje fuera de rango alto bloque A

3 Conjunto llaves tres posiciones - Alta: forzar carga - Media: Posición normal de trabajo - Baja: Reseteo bloque A

4 Conjunto leds indicación voltaje fuera de rango seteado bajo bloque A

5 Voltímetro indicación máximo voltaje de carga admitido bloque A

6 Potenciómetro selección máximo voltaje de carga bloque A

7 Led indicación

8 Amperímetro para carga y descarga bloque A

9 Led indicación

10 Led indicación

11 Voltímetro indicación mínimo voltaje de descarga admitido bloque A

12 Potenciómetro selección mínimo voltaje de descarga bloque A

13 Pulsador comienzo de carga bloque A

14 Display seteos bloque A y B

15 Pulsador visualización de seteos - selección de bloque - corriente - tiempo de ambos bloques

16 Pulsador aprobación seteos

17 Pulsador detención de actividad

18 Pulsador comienzo de descarga

19 Selector de bloques A - B o ambos

20 Selector automático/manual

21 Selector corriente (amp) para carga y descarga

22 Voltímetro indicación máximo voltaje de carga admitido bloque B

23 Potenciómetro selección máximo voltaje de carga bloque B

24 Led indicación

25 Voltímetro indicación mínimo voltaje de carga admitido bloque B

26 Potenciómetro selección mínimo voltaje de descarga bloque B

27 Amperímetro para carga y descarga boque B

28 Pulsador de puesta en marcha del equipo

29 Pulsador reseteo y apagado del equipo

30 Conjunto voltímetro bloque $\mathrm{B}$

31 Conjunto leds indicación voltaje fuera de rango alto bloque $\mathrm{B}$

32 Conjunto llaves tres posiciones - Alta: forzar carga - Media: Posición normal de trabajo - Baja: Reseteo bloque B

33 Conjunto leds indicación voltaje fuera de rango seteado bajo bloque B 


\section{Pruebas}

Si nota que su híbrido tiene menos energía que antes, que no responde como solía hacerlo, las capacidades de su batería pueden estar bajo algún tipo de falla o deterioro ya que necesita que la batería esté en óptimas condiciones para transferir toda esa energía a la tracción eléctrica. Si su paquete de batería no proporciona la cantidad adecuada de energía sentirá el vehículo más pesado o más lento.

Es por eso que la recuperación de la batería está ganando mucha popularidad. Los propietarios de vehículos híbridos pueden disfrutar de inmediato del rendimiento de su unidad nuevamente. El proceso de recuperación de la batería híbrida es sencillo, aunque largo y cuesta una fracción del costo de una batería nueva. Para hacer una diferencia en el medio ambiente, como ha sido el propósito de la mayoría de los conductores de vehículos híbridos, ahora la recuperación de la batería híbrida puede ayudar a eliminar los desechos en los basureros.

La batería del Toyota Highlander, por ejemplo, consta de 30 celdas individuales y cuando hay un problema con la batería, lo más probable es que se deba a una de las celdas, no a todos. Cada uno de las 30 celdas tiene su propio estado de salud y en un momento dado pueden estar en un estado diferente. Con el tiempo, los módulos en peor estado de este paquete terminan activando un código de fallas y eso a veces puede obligar a un distribuidor o un taller a reemplazar la batería completa. Sin embargo, es posible que uno o dos de los módulos deban reemplazarse y que no todo el paquete o la batería este en mal estado. El proceso de recuperación significa que el paquete se recicla extrayendo y reemplazando las celdas en mal estado.

\section{Proceso de recuperación de las baterías de alta tensión}

\section{Diagnóstico inicial}

El diagnóstico inicia cuando encontramos algún código de falla en el tablero que indique error o avería en el sistema de alta tensión, lo cual probablemente podría ser la batería. Para verificar se inicia el proceso con ayuda de un escáner, como el Techstream. No olvide ingresar los datos correctos del automóvil para asegurar una correcta conexión.

\section{Desmontaje de la batería}

Luego verificar con el escáner la existencia de un código de falla referente a la batería se procede a desmontar la batería de alta tensión que usualmente se encuentra ubicada por debajo del asiento posterior, para esto no olvide tomar las precauciones con el circuito de alto voltaje para evitar futuros contratiempos. 


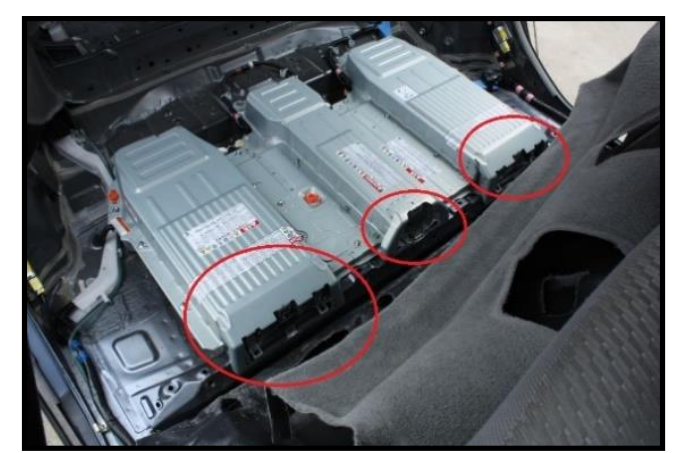

Figura 8. Proceso de extracción de la batería (Hybrid Automotive, 2016)

Proceso de descarga de la batería

1. Armamos dos bloques de 7 celdas cada uno ya que el equipo que utilizamos solo permite hasta un máximo de 14 celdas.

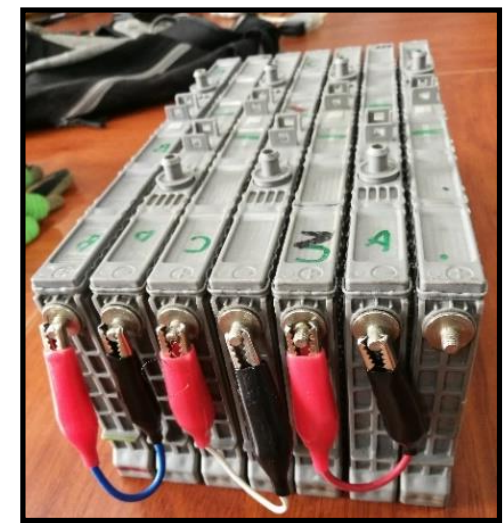

Figura 9. Conexión en serie de celdas

(Autores, 2018)

2. Realizamos la conexión a través de los terminales del banco hacia cada una de las celdas

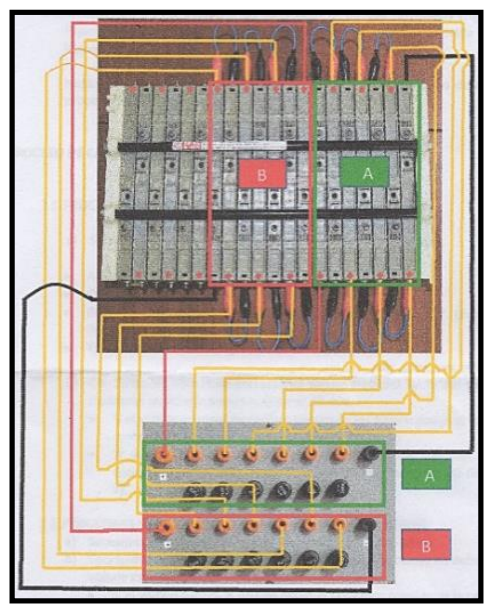

Figura 10. Diagrama de conexión

(TAAET Electronics , 2017)

3. Seteamos en el panel de control del banco 
$\checkmark$ Selecciona automático (\#20 todos los componentes)

$\checkmark$ Selecciona corriente de descarga 1 amp (\#21 todos los componentes)

$\checkmark$ Selecciona el voltaje mínimo con el potenciómetro de selección (\#12 y \#26 todos los componentes)

$\checkmark$ Selecciona el tiempo de 2 horas de descarga (\#15 todos los componentes)

Nota: el sistema de descarga automática se detendrá al momento que cualquiera de sus celdas llega al mínimo seleccionado de 6 voltios

4. Procedemos a descargar con los interruptores de tres posiciones (\#3 y \#32 todos los componentes) y descargamos cada celda hasta que llegue a 5 voltios

Proceso de carga de la batería

Proceso de precarga

1. Programamos en el panel de control del banco 30 min - 1 Amp.

$\checkmark$ Selecciona automático (\#20 todos los componentes)

$\checkmark$ Selecciona corriente de carga 1 Amp

$\checkmark$ Selecciona el máximo de voltaje en 9 v con el potenciómetro (\#6 y \#23 todos los componentes)

$\checkmark$ Selecciona el tiempo de 30 min (\#15 todos los componentes)

2. Concluido los 30 min de pre carga, el voltaje en todas las celdas tendría que mantener su voltaje nominal. Esperar durante 10 minutos.

$\checkmark$ Si el voltaje nominal no cambia dentro de los 10 minutos podemos consideras como celda aprobada en el primer procedimiento.

$\checkmark$ Si el voltaje nominal disminuye transcurriendo este tiempo podemos consideras como celda sospechosa para el cambio.

\section{Procedimiento de carga a una capacidad del $30 \%$}

1. Programamos en el panel de control del banco 60 min - 2 Amp.

2. Concluido los 60 min de carga, el voltaje en todas las celdas no debe pasar el voltaje máximo.

3. Si el voltaje supera el voltaje máximo podemos considerar la celda como sospechosa para el cambio.

\section{Proceso de descarga}

1. Configuramos en el panel de control una descarga a 1 Amp durante 2 horas

2. Mientras las celdas se están descargando las iremos categorizando tomando en cuenta el tiempo en que les toma llegar a su voltaje mínimo. Guiándonos por la siguiente tabla.

Tabla 4. Categorización de las celdas (TAAET Electronics , 2017)

\begin{tabular}{cc}
\hline Categoría & Tiempo (horas) \\
\hline $\mathbf{A}$ & $1: 41-2: 00$ \\
$\mathbf{B}$ & $1: 26-1: 40$ \\
\hline
\end{tabular}




\begin{tabular}{cc}
\hline $\mathbf{C}$ & $1: 10-1: 25$ \\
Celda defectuosa & Menos de 1:10 \\
\hline
\end{tabular}

\section{Resultados y discusión}

Luego de seguir el proceso de recuperación se obtuvo que de las 30 celdas que posee el Toyota Highlander, 3 de las mismas presentaban fallas, que según la categorización estaban en clase $\mathrm{C}$, lo que no permitía el correcto funcionamiento del vehículo.

Considerando que el techstream nos da la falla por bloques y siendo 2 celdas por bloque, se encontró la falla en bloque 3 y 4 del módulo LH de la batería de alta tensión.

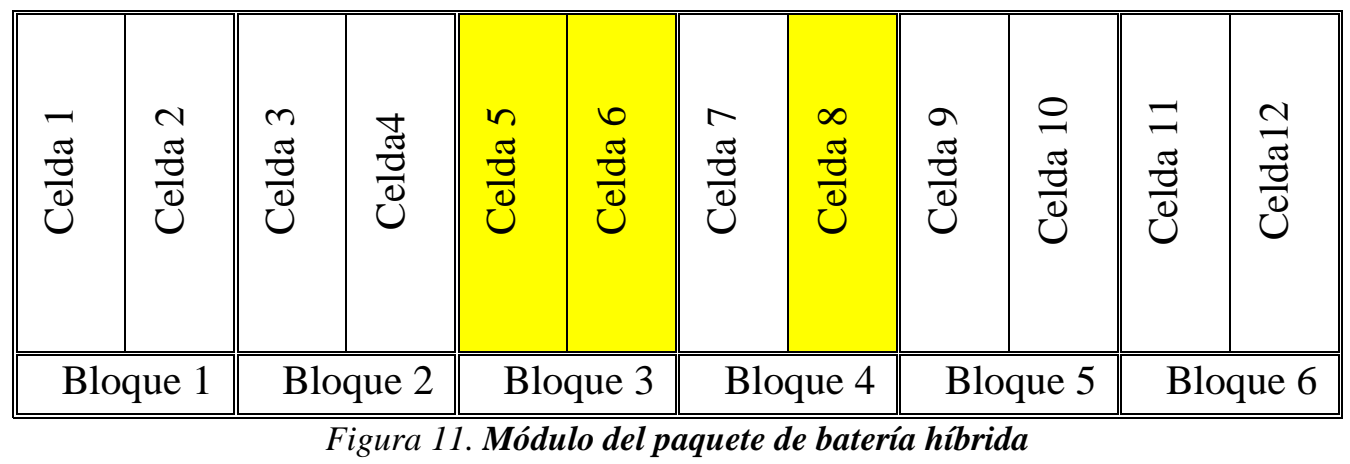

(Autores, 2018)

Siendo la celda 5, 6 y 8 las que presentaron fallas según el tiempo de descarga se las categorizadas tipo $\mathrm{C}$, mientras las demás estaban en categoría A por lo que solo es necesario el cambio de esas 3 celdas por otras de categoría A para volver a poner en buen funcionamiento el vehículo.

Tabla 5. Tiempo de descarga de las celdas en mal estado (Autores, 2018)

\begin{tabular}{cc}
\hline Celda & Tiempo (horas) \\
\hline $\mathbf{5}$ & $1: 12$ \\
$\mathbf{6}$ & $1: 16$ \\
$\mathbf{8}$ & $1: 15$ \\
\hline
\end{tabular}

Luego se coloca las nuevas celdas en reemplazo de las averidadas y lo más importante es realizar el proceso de balanceo de carga de todas las celdas antes de encender el vehículo, para que este no marque nuevos códigos de falla. El proceso de balanceo se reañiza de 8 a 12 horas aproximadamente.

Una vez intercambiadas estas tres celdas la batería de alta tensión volvió a estar en óptimas condiciones lo que conlleva que el funcionamiento del vehículo vuelva a ser el ideal.

\section{Conclusiones}


Viendo el crecimiento considerable de este mercado desde su aparición, el enfoque de los talleres hacia el mantenimiento y reparación de esta tecnología es completamente necesario ya que sin duda invadirá nuestro mercado con el pasar de los años. Por lo que la necesidad de especializarse y aprender de estos procedimientos permitirá obtener otro mercado.

Sin duda alguna el proceso de recuperación de batería es lo más conveniente al momento de considerar el gasto que representa reemplazar la batería completa, sin dejar de lado los beneficios medioambientales que representa reusar la batería que ya posee nuestro vehículo y solo reemplazar las celdas en mal estado.

El vehículo cuenta con diversos sistemas de seguridad además de la protección que el equipo personal debe tener, por lo que seguirlos sin evadir paso alguno es esencial siendo consiente de los altos voltajes que este sistema maneja.

\section{Bibliografía}

AEADE. (2017). Asociacion De Empresas Automotrices Del Ecuador. Obtenido de http://www.aeade.net/anuario-2016/anuario2016.pdf

Cars. (25 de 06 de 2014). Cars. Obtenido de https://www.cars.com/research/toyotahighlander_hybrid-2014/

Comercio, R. L.-E. (2013). Revista Líderes. Obtenido de http://www.revistalideres.ec/lideres/hyundai-le-apuesta-hibrido-pais.html

Curtis Anderson, J. A. (2010). Electric and Hybrid Cars. Mcfarland.

Ecolectric Battery - (23 de 05 de 2017). Ecolectric Battery . Obtenido de http://ecolectricbattery.com/1/post/2017/05/modelos-de-vehiculos-hibridos-guia.html

Hybrid Automotive. (2016). Hybrid Automotive. Obtenido de https://hybridautomotive.com/pages/install-hh1

Liker, J. K. (2006). Japanese automakers, US suppliers and supply chain superiority. Supply Chains and Total Product Systems: A Reader.

Motor trend. (01 de 03 de 2008). Motor trend. Obtenido de http://www.motortrend.com/cars/toyota/highlander/2008/

Pèrez, J. (14 de 02 de 2017). Motor Zeta. Obtenido de https://www.elperiodico.com/es/motor/quienes-somos.shtml

Sánchez, M. \&. (2017). Análisis, diagnóstico y propuesta de mejora para una cadena de suministro de autopartes en el Perú utilizando el modelo SCOR.

TAAET Electronics . (2017). Manual de uso del banco de pruebas de bateria hibrida. Guayaquil. 
Toyota. (2008). Emergency response guide. Toyota motor company.

Toyota. (2015). Blog Toyota. Obtenido de http://blog.toyota.co.uk/how-does-toyota-hybridsynergy-drive-work

Toyota. (2018). Toyota de Puerto Rico, Corp. Obtenido de http://www.toyotapr.com/hibridos 